\title{
Analytic evaluation of Franck-Condon integrals for anharmonic vibrational wave functions
}

\author{
M. Carvajal, J. M. Arias, and J. Gómez-Camacho \\ Departamento de Física Atómica, Molecular y Nuclear, Facultad de Física, Universidad de Sevilla, Apartado 1065, \\ 41080 Sevilla, Spain \\ (Received 11 December 1998)
}

\begin{abstract}
The problem of calculating Franck-Condon overlap integrals in molecular transitions between vibrational states in different electronic configurations is addressed. An exact and easily applicable analytical expression is obtained when the vibrational states can be approximated by eigenstates of Morse potentials with different strengths and locations but identical ranges. An approximate analytical expression is obtained for the general case. The method is applied to the stretching S-S mode corresponding to $\widetilde{C}^{1} v_{2} \leftarrow \widetilde{X}^{1} v_{1}$ transitions in the $\mathrm{S}_{2} \mathrm{O}$ molecule. [S1050-2947(99)09505-0]

PACS number(s): 33.70.Ca, 31.15.-p, 02.60.-x
\end{abstract}

\section{INTRODUCTION}

Molecular Franck-Condon factors [1] describe the dependence on the vibrational wave functions of vibronic transitions, which are transitions between vibrational states in different electronic configurations. Assuming that the electronic transition moment is independent of the location of the nuclei, the Franck-Condon factors are the square of the overlap integral between the initial and final vibrational wave functions in the vibronic transition. The Franck-Condon factors give the molecular structure information needed to evaluate the band intensities in emission and absorption. During the past few years the improvement in experimental techniques has opened the possibility of measuring with reasonable resolution multidimensional Franck-Condon factors for transitions between vibronic levels differing in up to around 20 quanta [2]. These new data are a challenge for the theory. On one side, the treatment of polyatomic molecules introduces important complications in the theoretical description of the corresponding multidimensional potential energy surface. Those should be solved in order to understand the existing data and produce accurate predictions for new experiments. On the other hand, as the transitions measured populate vibrational states with high excitation energy in a given potential energy surface, one can explore the effect of anharmonicity.

Up to now most of the work done along this line relies on the simplification of assuming simple harmonic oscillator molecular potentials for the two electronic states involved in the transition [2-9]. This approximation, which has been proved to be sufficiently accurate for describing the data involving few states around the bottom of the potential energy surfaces, is expected to be poorer as one goes higher in excitation energy and consequently explores regions where the anharmonicity becomes important. A treatment in which the nuclear motion is governed by anharmonic potentials seems to be in order. However, the treatment of the Morse [10] or the Pöschl-Teller [11] anharmonic potentials, which are the most popular ones, do not provide in general, up to now, simple analytical expressions. In the case of the overlap integrals between states of Morse potentials with different strengths and locations but identical ranges, an analytical expression has been recently presented [12]. However, that ex- pression, as it is mentioned in that reference, is not very useful for applications to molecules because it has an alternating series which becomes unstable for realistic strengths, due to computational precision errors. In view of these problems, an approximate treatment of anharmonicity has been proposed that uses modified harmonic-oscillator wave functions $[2,12]$. However it should be noted that these states are not orthogonal because the oscillator parameter depends on the vibrational quantum number.

It is worth mentioning that direct integration of the overlap integrals is difficult as far as highly excited states in the Morse potentials are concerned. Computational errors in the evaluation of the integrals can appear since one has to integrate two rapidly oscillating functions. Nevertheless, effort has been devoted to the correct numerical solution of the corresponding Schrödinger equation for a Morse potential by several methods [13].

In this paper we present an analytic formulation for the overlap Franck-Condon integrals between states of Morse potentials. This treatment is based on the idea of configuration localized Morse states recently introduced [14] and it is closely related to the concept of quadrature in Gaussian integration. We deduce an exact analytic formula for Morse potentials with different strengths and locations but identical ranges. We demonstrate that the expression deduced here is equivalent to the one given in Ref. [12], but in our presentation applications to realistic molecules are easy and there are no problems with possible numerical errors. In addition, an approximate analytic expression for the case of arbitrary vibrational wave functions is also obtained.

The paper is structured as follows. In Sec. II, we revise briefly the recently introduced idea of configuration localized Morse (CLM) states and the integration by quadratures in the Gauss method. In Sec. III we apply those concepts to the calculation of overlap Franck-Condon integrals. In Sec. IV, some applications of the results obtained in the preceding section are shown. Finally, the summary and conclusions are presented in Sec. V.

\section{CONFIGURATION LOCALIZED MORSE STATES AND GAUSSIAN INTEGRATION BY QUADRATURES}

In this section we discuss briefly the concepts of configuration localized Morse (CLM) states and the method of 
Gauss integration by quadratures. These are the two ingredients on which the derivation of the analytic expressions of the Franck-Condon overlap integrals presented in the next section relies.

The Morse potential is

$$
V(r)=D\left(\{1-\exp [-\beta(r-R)]\}^{2}-1\right),
$$

where $-D$ is the value of the potential in its minimum, $R$ is the equilibrium position, and $\beta$ is the inverse of the range of the potential. Since the Morse Hamiltonian is related to the su(2) algebra [15], its bound states can be labeled by $|j m\rangle$. The quantum number $j$ is an integer related to the depth of the potential while $m$ is related to the binding energy of the state,

$$
D=\frac{\hbar^{2} \beta^{2}}{2 \mu}\left(j+\frac{1}{2}\right)^{2}, \quad \epsilon_{m}=-\frac{\hbar^{2} \beta^{2}}{2 \mu} m^{2},
$$

where $\mu$ is the reduced mass of the system. The label $m$ can take the values $m=j, j-1, \ldots, 1$ (the value $m=0$ is excluded since it corresponds to a state which is not normalizable and will not be considered in the rest of this paper). The state most tightly bound corresponds to $m=j$. With this notation the bound eigenstates of the Morse Hamiltonian are written as

$$
\langle r \mid j m\rangle=\Psi_{j m}(r)=\mathcal{N}_{j m}^{-1 / 2} \beta^{1 / 2} \exp \left[-\frac{y}{2}\right] y^{m} L_{j-m}^{(2 m)}(y),
$$

where $\mathcal{N}_{j m}$ is a normalization constant, $y=(2 j$ $+1) \exp [-\beta(r-R)]$ is the Morse variable, and $L_{s}^{(p)}(y)$ are the generalized Laguerre polynomials of degree $s$ and order $p$.

\section{A. Configuration localized Morse (CLM) states}

The CLM states [14] are obtained from an orthogonal transformation of the Morse bound states (3) given by

$$
|j k(C L M)\rangle=\sum_{m=1}^{j}\langle j m \mid j k(C L M)\rangle|j m\rangle,
$$

where

$$
\begin{gathered}
\langle j m \mid j k(C L M)\rangle=N_{k}^{-1 / 2} \mathcal{N}_{j m}^{-1 / 2} P_{j-1}^{(m)}\left(y_{k}\right), \\
N_{k}=\frac{j+1}{y_{k}}\left[L_{j-1}^{(1)}\left(y_{k}\right)\right]^{2},
\end{gathered}
$$

$P_{j-1}^{(m)}(y)$ are polynomials of degree $j-1$ defined by

$$
P_{j-1}^{(m)}(y)=y^{m-1} L_{j-m}^{(2 m)}(y),
$$

and $y_{k}$ are the zeros of the polynomial $L_{j}^{(1)}(y)$. These CLM states are orthogonal and can be used for calculating matrix elements of any function of $r$. Its properties are discussed in Ref. [14], where it is shown that the CLM states are well suited to describe anharmonic effects in infrared transitions.

The CLM states can be written in coordinate representation as

$$
\langle r \mid j k(C L M)\rangle=\Psi_{j k}(r)=N_{k}^{-1 / 2} \beta^{1 / 2} y \exp \left[-\frac{y}{2}\right] Q_{j-1}^{(k)}(y),
$$

where $Q_{j-1}^{(k)}(y)$ are $(j-1)$-degree polynomials defined by

$$
Q_{j-1}^{(k)}(y)=L_{j-1}^{(1)}\left(y_{k}\right) \frac{L_{j}^{(1)}(y)}{y-y_{k}} .
$$

It is clear that $Q_{j-1}^{(k)}(y)$ vanishes when $y=y_{\ell}$ is a zero of $L_{j}^{(1)}(y)$ different from $y_{k}$. For the case $y=y_{k}$,

$$
N_{k}=Q_{j-1}^{(k)}\left(y_{k}\right) .
$$

Note that from the orthogonality of these states one gets the relation

$$
\int_{0}^{\infty} d y y \exp [-y] Q_{N-1}^{(k)}(y) Q_{N-1}^{(\ell)}(y)=Q_{N-1}^{(\varrho)}\left(y_{\ell}\right) \delta_{k, \ell} .
$$

\section{B. Gaussian integration by quadratures}

Consider a family of orthogonal polynomials $P_{n}(x)$ with $n=1,2, \ldots$ ( $n$ is the polynomial degree) in an interval $a$ $\leqslant x \leqslant b$ with respect to a weight function $\omega(x)$,

$$
\int_{a}^{b} d x \omega(x) P_{n}(x) P_{m}(x)=h_{n} \delta_{n, m} .
$$

Let us call $x_{k}$, with $k=1,2, \ldots, N$, to the zeros of one of those polynomials $P_{N}(x)$. From them, $N$ polynomials of degree $N-1$ can be easily obtained,

$$
\mathcal{Q}_{N-1}^{(k)}(x)=\frac{P_{N-1}\left(x_{k}\right)}{h_{N-1}} \frac{k_{N-1}}{k_{N}} \frac{P_{N}(x)}{\left(x-x_{k}\right)},
$$

where $k_{N}$ is the coefficient of $x^{N}$ in $P_{N}(x)$. Note that Eq. (13) is formally equivalent to Eq. (9). These polynomials are orthogonal in the same interval and relative to the same weight function as the original polynomials,

$$
\int_{a}^{b} d x \omega(x) \mathcal{Q}_{N-1}^{(k)}(x) \mathcal{Q}_{N-1}^{(\varrho)}(x)=\mathcal{Q}_{N-1}^{(\varrho)}\left(x_{\ell}\right) \delta_{k, \ell} .
$$

Note that Eq. (11) is a particular case of this equation for $\omega(x)=x \exp [-x]$

In the next section we are interested in evaluating integrals of the type

$$
\int_{a}^{b} d x \omega(x) F(x)
$$

where $\omega(x)$ is a weight function and $F(x)$ is any function of $x . F(x)$ can be written in the interval $(a, b)$ as an expansion in terms of quadratic forms of orthogonal polynomials $\left[P_{n}(x)\right]$ associated with the same weight function. Ignoring terms of the order of the $2 N+1$ derivative of $F(x)$, we can write

$$
F(x) \simeq \sum_{n, m=0}^{N} C_{n m} P_{n}(x) P_{m}(x) .
$$


It should be noted that the term $(N N)$ is the only one contributing to the $2 N$ derivative of $F(x)$. Thus, the $2 N$ derivative of $F(x)$ is written as

$$
F^{(2 N)}(x) \simeq C_{N N} k_{N}^{2}(2 N) ! .
$$

The polynomials $\left\{P_{n}(x) ; n=0, \ldots, N-1\right\}$ can be expanded in terms of the new set of orthogonal polynomials $\left\{\mathcal{Q}_{N-1}^{(k)}(x) ; k=1, \ldots, N\right\}$ defined in Eq. (13). Thus, up to the same order as before, $F(x)$ can be written as

$$
\begin{aligned}
F(x) \simeq & \sum_{k, \ell=1}^{N} B_{k \ell} \mathcal{Q}_{N-1}^{(k)}(x) \mathcal{Q}_{N-1}^{(\ell)}(x) \\
& +\sum_{k=1}^{N} B_{k}^{\prime} \mathcal{Q}_{N-1}^{(k)}(x) P_{N}(x)+C_{N N} P_{N}(x) P_{N}(x) .
\end{aligned}
$$

If now we compute the integral in Eq. (15), taking into account Eqs. (18) and (14),

$$
\int_{a}^{b} d x \omega(x) F(x) \simeq \sum_{k=1}^{N} B_{k k} \mathcal{Q}_{N-1}^{(k)}\left(x_{k}\right)+C_{N N} h_{N} .
$$

The coefficients $B_{k k}$ can be evaluated, ignoring corrections of the order of the $2 N+1$ derivative of $F(x)$ from Eq. (18),

$$
F\left(x_{k}\right)=B_{k k}\left[\mathcal{Q}_{N-1}^{(k)}\left(x_{k}\right)\right]^{2} .
$$

Thus, finally, we obtain

$$
\int_{a}^{b} d x \omega(x) F(x)=\sum_{k=1}^{N} F\left(x_{k}\right) \omega_{k}+R_{N},
$$

where the sum is extended to the zeros of the polynomial $P_{N}(x), F\left(x_{k}\right)$ is the function evaluated at those zeros, and

$$
\omega_{k}=\left[\mathcal{Q}_{N-1}^{(k)}\left(x_{k}\right)\right]^{-1} \text {. }
$$

The residual $R_{N}$ can be easily evaluated to be

$$
R_{N} \simeq \frac{h_{N}}{k_{N}^{2}(2 N) !} F^{(2 N)}(\xi), \quad a<\xi<b .
$$

Equation (21) will be of use in the next section.

In the particular case in which the interval is $(0, \infty)$ and the weight function $\omega(y)=y^{\alpha} \exp [-y]$, the appropriate polynomials $P_{N}(x)$ are the generalized Laguerre polynomials of order $\alpha, L_{N}^{(\alpha)}(y)$. The application of Eqs. (21) and (22) to the case of the generalized Laguerre polynomials can be obtained directly with $k_{N} / k_{N-1}=-N^{-1}, \quad h_{N-1}=(N+\alpha)$ !/ $(N-1)$ !, $P_{N-1}(y)=L_{N-1}^{(\alpha)}(y)$, and using the known relation [16] (Eq. 22.8.6)

$$
y\left[L_{N}^{(\alpha)}(y)\right]^{\prime}=N\left[L_{N}^{(\alpha)}(y)\right]-(N+\alpha)\left[L_{N-1}^{(\alpha)}(y)\right] .
$$

With these expressions, Eq. (13) and Eq. (22), we obtain

$$
\omega_{k}=\frac{(N+\alpha) !}{N !} \frac{y_{k}}{(N+\alpha)^{2}\left[L_{N-1}^{(\alpha)}\left(y_{k}\right)\right]^{2}} .
$$

It should be noted that in the particular case of $\alpha=0$ listed in Ref. [16] (Equation 25.4.45) there is an erratum since an $N$ ! is written in the numerator which should not be there. In the next section the case of $\alpha=1$ will be of special interest. In that case, $\mathcal{Q}_{N-1}^{(k)}\left(y_{k}\right)$ are precisely the $Q_{N-1}^{(k)}\left(y_{k}\right)$ introduced in the preceding subsection, Eqs. (9) and (10), in relation to the CLM,

$$
\mathcal{Q}_{j-1}^{(k)}\left(y_{k}\right)=Q_{j-1}^{(k)}\left(y_{k}\right)=N_{k},
$$

and then

$$
\omega_{k}=N_{k}^{-1}
$$

\section{FRANCK-CONDON OVERLAP INTEGRALS}

In this section we discuss the general problem of calculating the overlap integral of two wave functions. The integral that we want to evaluate is

$$
I=\int_{r(\min )}^{r(\max )} d r \Phi_{\alpha_{1}}^{(1) *}(r) \Phi_{\alpha_{2}}^{(2)}(r)
$$

where the label $\alpha$ includes all the quantum numbers needed to identify the vibrational state. The superindex (1) or (2) identifies the electronic state. Here $r$ indicates the coordinate that describes the vibrational excitation. It will be the interatomic separation for stretching modes but it may correspond to angles for a bending mode. Let us introduce a new variable $y(r)$ which is a smooth function of $r$ with the proper asymptotic behavior: $y(r) \rightarrow 0$ if $r \rightarrow r(\max )$ and $y(r) \rightarrow \infty$ if $r \rightarrow r(\min )$. Then, the preceding equation is

$$
I=\int_{0}^{\infty} d y \frac{1}{\left(-\frac{d y}{d r}\right)} \Phi_{\alpha_{1}}^{(1) *}(r(y)) \Phi_{\alpha_{2}}^{(2)}(r(y)) .
$$

This integral can be rewritten as

$$
I=\int_{0}^{\infty} d y y \exp [-y]\left[\frac{\exp [y]}{\left(-\frac{d y}{d r}\right)} \frac{1}{y} \Phi_{\alpha_{1}}^{(1) *}(r(y)) \Phi_{\alpha_{2}}^{(2)}(r(y))\right] .
$$

This integral can be evaluated approximately by Gaussian integration with $n$ points, where $n$ is a large number. By using Eqs. (21) and (27) we obtain

$$
I \approx \sum_{k=1}^{n} \frac{1}{N_{k}}\left[\frac{\exp \left[y_{k}\right]}{\left(-\frac{d y}{d r}\right)_{y_{k}}} \frac{1}{y_{k}} \Phi_{\alpha_{1}}^{(1) *}\left(r\left(y_{k}\right)\right) \Phi_{\alpha_{2}}^{(2)}\left(r\left(y_{k}\right)\right)\right]
$$

where the sum is extended to the zeros of the generalized Laguerre polynomials of order $1, L_{n}^{(1)}(y)$. The approximation is better as $n$ gets larger. It should be noticed that this approximation can be applied in the case in which the electronic dipole matrix element is considered to be a function of the interatomic separation. Then, 


$$
\begin{aligned}
I^{\prime}= & \int_{r(\min )}^{r(\max )} d r \Phi_{\alpha_{1}}^{(1) *}(r) \Phi_{\alpha_{2}}^{(2)}(r) \mu(r) \\
\approx & \sum_{k=1}^{n} \frac{1}{N_{k}}\left[\frac{\exp \left[y_{k}\right]}{\left(-\frac{d y}{d r}\right)_{y_{k}}} \frac{1}{y_{k}} \Phi_{\alpha_{1}}^{(1) *}\left(r\left(y_{k}\right)\right)\right. \\
& \left.\times \Phi_{\alpha_{2}}^{(2)}\left(r\left(y_{k}\right)\right) \mu\left(r\left(y_{k}\right)\right)\right] .
\end{aligned}
$$

Let us now consider the following set of orthogonal states:

$$
\begin{aligned}
\langle r \mid n k(C L M)\rangle & =\Psi_{n k}(r) \\
& =N_{k}^{-1 / 2}\left(-\frac{d y}{d r}\right)^{1 / 2}\left(\frac{1}{y}\right)^{1 / 2} y \exp \left[-\frac{y}{2}\right] Q_{n-1}^{(k)}(y) .
\end{aligned}
$$

These are formally equivalent to the CLM states discussed before. Note that if $y=(2 n+1) \exp \left[-\beta\left(r-R_{0}\right)\right]$, then they will just be CLM states of a Morse potential with $n$ bound states, Eq. (8). The overlap of these states with the original states in Eq. (29) can be calculated by Gaussian integration to be

$$
\begin{aligned}
& \left\langle n k(C L M) \mid \Phi_{\alpha_{1}}^{(1)}\right\rangle \\
& \quad=N_{k}^{-1 / 2} \frac{1}{\left(-\frac{d y}{d r}\right)_{y_{k}}^{1 / 2}}\left(\frac{1}{y_{k}}\right)^{1 / 2} \exp \left[\frac{y_{k}}{2}\right] \Phi_{\alpha_{1}}^{(1) *}\left(r\left(y_{k}\right)\right),
\end{aligned}
$$

and similarly for $\Phi_{\alpha_{2}}^{(2)}$. Thus, Eq. (31) can be written as

$$
I \approx \sum_{k=1}^{n}\left\langle\Phi_{\alpha_{1}}^{(1)} \mid n k(C L M)\right\rangle\left\langle n k(C L M) \mid \Phi_{\alpha_{2}}^{(2)}\right\rangle
$$

Consequently, the overlap integral of a product of arbitrary functions can be written in terms of a product of overlaps of these functions with a basis of states which are formally equivalent to CLM states. Moreover, we can choose the function $y(r)$ in Eq. (30) so that the expression in brackets can be approximated by a polynomial of order $(2 n-1)$ in the variable $y$. If so, the sum can be understood as a projection on the space generated by $n$ CLM states. If there are $j_{1}$ vibrational wave functions $\Phi_{\alpha_{1}}^{(1)}(r)$ and $j_{2}$ vibrational wave functions $\Phi_{\alpha_{2}}^{(2)}(r)$, then a polynomial approximation to its product will require polynomials of order at least $\left(j_{1}+j_{2}\right.$ $-2)$. Then, taking $n$ as the closer integer to $\left(j_{1}+j_{2}\right) / 2$, the overlap integral can be approximated by a sum of $n$ terms.

Now we will apply the general ideas discussed above to the case of Morse wave functions corresponding to different Morse potentials [in general, different strengths $(j)$, locations $(R)$, and ranges $(\beta)]$,

$$
I=\int_{-\infty}^{\infty} d r \Psi_{j_{1} m_{1}}^{*}\left(\beta_{1}, R_{1} ; r\right) \Psi_{j_{2} m_{2}}\left(\beta_{2}, R_{2} ; r\right) .
$$

If one considers the form of the Morse wave functions (3) in terms of the polynomials $P_{j-1}^{(m)}$ defined in Eq. (7), introduces the Morse variables associated to each Morse potential

$$
\begin{aligned}
& y_{1}=\left(2 j_{1}+1\right) \exp \left[-\beta_{1}\left(r-R_{1}\right)\right], \\
& y_{2}=\left(2 j_{2}+1\right) \exp \left[-\beta_{2}\left(r-R_{2}\right)\right],
\end{aligned}
$$

and defines the new variable $y(r)$ as stated in Eq. (29), the original integral can be rewritten as

$$
\begin{aligned}
I= & \mathcal{N}_{j_{1} m_{1}}^{-1 / 2} \sqrt{\beta_{1}} \mathcal{N}_{j_{2} m_{2}}^{-1 / 2} \sqrt{\beta_{2}} \int_{0}^{\infty} d y y \exp [-y]\left[\frac{y_{1} y_{2}}{y\left(-\frac{d y}{d r}\right)}\right. \\
& \left.\times \exp \left(y-\frac{y_{1}+y_{2}}{2}\right) P_{j_{1}-1}^{\left(m_{1}\right)}\left(y_{1}\right) P_{j_{2}-1}^{\left(m_{2}\right)}\left(y_{2}\right)\right]
\end{aligned}
$$

Then, Eq. (31) gives in this case

$$
\begin{aligned}
I \approx & \mathcal{N}_{j_{1} m_{1}}^{-1 / 2} \sqrt{\beta_{1}} \mathcal{N}_{j_{2} m_{2}}^{-1 / 2} \sqrt{\beta_{2}} \sum_{k=1}^{j} N_{k}^{-1} \frac{\left(y_{1}\right)_{k}\left(y_{2}\right)_{k}}{y_{k}\left(-\frac{d y}{d r}\right)_{k}} \\
& \times \exp \left[y_{k}-\frac{\left(y_{1}\right)_{k}+\left(y_{2}\right)_{k}}{2}\right] \\
& \times P_{j_{1}-1}^{\left(m_{1}\right)}\left(\left(y_{1}\right)_{k}\right) P_{j_{2}-1}^{\left(m_{2}\right)}\left(\left(y_{2}\right)_{k}\right) .
\end{aligned}
$$

This is the final expression in which $\mathcal{N}_{j m}$ are the normalization constants of the Morse wave functions, the sum is extended to the number of zeros of the polynomial $L_{j}^{(1)}(y)$ (an appropriate election of $j$ is $\left[\left(j_{1}+j_{2}\right) / 2\right]$, as justified above), and $\left(y_{1}\right)_{k}$ and $\left(y_{2}\right)_{k}$ are the values obtained for $y_{1}$ and $y_{2}$ with the value $r=r_{k}, r_{k}$ obtained from $y\left(r_{k}\right)=y_{k}$.

The choice of the function $y(r)$ is arbitrary, but it should be chosen so that the expression in brackets in Eq. (38) can be approximated by a polynomial. Taking $y=\left(y_{1}+y_{2}\right) / 2$, the exponential in Eq. (39) is 1, leading to the expression

$$
\begin{aligned}
I \approx & \mathcal{N}_{j_{1} m_{1}}^{-1 / 2} \sqrt{\beta_{1}} \mathcal{N}_{j_{2} m_{2}}^{-1 / 2} \sqrt{\beta_{2}} \\
& \times \sum_{k=1}^{j} N_{k}^{-1} \frac{2\left(y_{1}\right)_{k}\left(y_{2}\right)_{k}}{y_{k}} \frac{1}{\beta_{1}\left(y_{1}\right)_{k}+\beta_{2}\left(y_{2}\right)_{k}} \\
& \times P_{j_{1}-1}^{\left(m_{1}\right)}\left(\left(y_{1}\right)_{k}\right) P_{j_{2}-1}^{\left(m_{2}\right)}\left(\left(y_{2}\right)_{k}\right) .
\end{aligned}
$$

It should be noted that there is room to investigate whether alternative choices of the function $y(r)$ could improve the calculation of the integral $I$.

Expression (40) is approximate in general. We will show now that it becomes exact for the case of $\beta_{1}=\beta_{2}$ if $j$ is taken as $j=\left(j_{1}+j_{2}\right) / 2$ or larger. The exact evaluation of that case can be done by using the method of Gaussian integration by quadratures sketched in the preceding section. In this case the integral to be done is 
TABLE I. Franck-Condon matrix elements between states in identical Morse potentials but displaced one respect to the other, $\left\langle j_{1} m_{1} \mid j_{2} m_{2}\right\rangle: j_{1}=j_{2}=5, \beta_{1}=\beta_{2}=0.90 \AA^{-1}, R_{1}=2.67 \AA$, and $R_{2}=3.60 \AA$. Successive entries correspond to the exact calculation making the integration of the Morse wave functions (first line), which is identical to our results given in Eq. (46), the calculation using harmonic oscillator wave functions with oscillator length $a_{0}=\left[\left(j+\frac{1}{2}\right)^{1 / 2} \beta\right]^{-1}$ (second line), and the calculation with modified harmonic-oscillator wave functions as given in Ref. [12] (third line).

\begin{tabular}{|c|c|c|c|c|c|}
\hline$m_{1} \backslash m_{2}$ & 5 & 4 & 3 & 2 & 1 \\
\hline \multirow[t]{3}{*}{5} & 0.42687 & -0.34228 & 0.27839 & -0.21679 & 0.14889 \\
\hline & 0.38163 & -0.52971 & 0.51990 & -0.41663 & 0.28915 \\
\hline & 0.38163 & -0.45147 & 0.49396 & -0.52002 & 0.50695 \\
\hline \multirow[t]{3}{*}{4} & 0.79048 & -0.12775 & 0.04693 & -0.00110 & -0.01166 \\
\hline & 0.52971 & -0.35362 & -0.02750 & 0.32220 & -0.43193 \\
\hline & 0.62781 & -0.28358 & 0.05443 & 0.16330 & -0.38378 \\
\hline \multirow[t]{3}{*}{3} & 0.41678 & 0.74799 & -0.13014 & 0.13408 & -0.08036 \\
\hline & 0.51990 & 0.02750 & -0.38061 & 0.28255 & 0.03174 \\
\hline & 0.23717 & 0.51298 & -0.40591 & 0.24204 & 0.01297 \\
\hline \multirow[t]{3}{*}{2} & -0.12667 & 0.44613 & 0.77692 & 0.04596 & 0.09256 \\
\hline & 0.41663 & 0.32220 & -0.28255 & -0.15417 & 0.35170 \\
\hline & -0.09261 & 0.32338 & 0.41678 & -0.40496 & 0.29047 \\
\hline \multirow[t]{3}{*}{1} & 0.02807 & -0.24277 & 0.21095 & 0.81696 & 0.36737 \\
\hline & 0.28915 & 0.43193 & 0.03174 & -0.35170 & 0.08991 \\
\hline & 0.05862 & -0.19434 & 0.36082 & 0.38247 & -0.39483 \\
\hline
\end{tabular}

$$
\begin{aligned}
I= & \int_{-\infty}^{\infty} d r \Psi_{j_{1} m_{1}}^{*}\left(\beta, R_{1} ; r\right) \Psi_{j_{2} m_{2}}\left(\beta, R_{2} ; r\right) \\
= & \mathcal{N}_{j_{1} m_{1}}^{-1 / 2} \mathcal{N}_{j_{2} m_{2}}^{-1 / 2} \beta \int_{-\infty}^{\infty} d r y_{1} y_{2} \\
& \times \exp \left[\frac{y_{1}+y_{2}}{2}\right] P_{j_{1}-1}^{\left(m_{1}\right)}\left(y_{1}\right) P_{j_{2}-1}^{\left(m_{2}\right)}\left(y_{2}\right),
\end{aligned}
$$

where we have used Eqs. (3) and (7) and have introduced the Morse variables

$$
\begin{aligned}
& y_{1}=\left(2 j_{1}+1\right) \exp \left[-\beta\left(r-R_{1}\right)\right]=c_{1} \exp [-\beta r], \\
& y_{2}=\left(2 j_{2}+1\right) \exp \left[-\beta\left(r-R_{2}\right)\right]=c_{2} \exp [-\beta r] .
\end{aligned}
$$

Introducing a new variable $y=\left(y_{1}+y_{2}\right) / 2$, we have

$$
y=\frac{c_{1}+c_{2}}{2} \exp [-\beta r]=c \exp [-\beta r]
$$

In terms of this new variable Eq. (41) can be rewritten as

$$
\begin{aligned}
I= & \mathcal{N}_{j_{1} m_{1}}^{-1 / 2} \mathcal{N}_{j_{2} m_{2}}^{-1 / 2} \frac{c_{1} c_{2}}{c^{2}} \int_{0}^{\infty} d y y \\
& \times \exp [-y] P_{j_{1}-1}^{\left(m_{1}\right)}\left(\frac{c_{1}}{c} y\right) P_{j_{2}-1}^{\left(m_{2}\right)}\left(\frac{c_{2}}{c} y\right) .
\end{aligned}
$$

This last integral can be evaluated by quadratures by using the orthogonal polynomials in the interval $(0, \infty)$ with weight function $\omega(y)=y \exp [-y]$. In our case $P_{j_{1}-1}^{\left(m_{1}\right)}\left(\left(c_{1} / c\right) y\right) P_{j_{2}-1}^{\left(m_{2}\right)}\left(\left(c_{2} / c\right) y\right)$ is a polynomial of order $j_{1}$ $+j_{2}-2$. Then, if we take $j=\left[\left(j_{1}+j_{2}\right) / 2\right]$ so that $R_{N}$ in Eq. (23) is zero, the result of the quadrature is exact and gives [see Eq. (27)]

$$
I=\mathcal{N}_{j_{1} m_{1}}^{-1 / 2} \mathcal{N}_{j_{2} m_{2}}^{-1 / 2} \frac{c_{1} c_{2}}{c^{2}} \sum_{k=1}^{j} N_{k}^{-1} P_{j_{1}-1}^{\left(m_{1}\right)}\left(\frac{c_{1}}{c} y_{k}\right) P_{j_{2}-1}^{\left(m_{2}\right)}\left(\frac{c_{2}}{c} y_{k}\right),
$$

where we have used Eq. (6). Equation (46) is the exact result and it is precisely what is obtained from Eq. (40) for $\beta_{1}$ $=\beta_{2}$. It can be evaluated simply since it involves the sum of products of polynomials evaluated at the zeros of other polynomials [in this case the generalized Laguerre polynomials of order $\left.1, L_{j}^{(1)}(y)\right]$. In the appendix it is shown that this expression is equivalent to Eq. (4.5) in Ref. [12], avoiding the problems with numerical errors.

\section{APPLICATIONS}

In order to test how good different approximations are for the evaluation of the Franck-Condon overlap integrals, we have performed a first calculation for a simple case in which the anharmonicity should be important. In Table I we show a calculation in which both electronic configurations involved in the vibronic transition give rise to identical Morse potentials in depth and strength but somehow displaced. We have chosen a small value for $j$ so as to see clearly the anharmonic effects. In this case $j_{1}=j_{2}=5, \beta_{1}=\beta_{2}=0.90 \AA^{-1}, R_{1}$ $=2.67 \AA$, and $R_{2}=3.60 \AA$. Different entries in the table for $m_{1}$ and $m_{2}$ fixed are the following. The first entry is the exact result obtained by direct integration of the corresponding Morse wave functions, which coincides with our analytic 
TABLE II. Franck-Condon matrix elements between states in different Morse potentials $\left\langle j_{1} m_{1} \mid j_{2} m_{2}\right\rangle$ : $j_{1}=5, R_{1}=2.67 \AA, \beta_{1}=0.90 \AA^{-1}$ and $j_{2}=5, R_{2}=3.60 \AA, \beta_{2}=0.60 \AA^{-1}$. Successive entries correspond to the exact calculation making the integration of the Morse wave functions (first line), our approximate results given by Eq. (40) (second line), the calculation using harmonic-oscillator wave functions with oscillator length $a_{0}=\left[\left(j+\frac{1}{2}\right)^{1 / 2} \beta\right]^{-1}$ (third line), and the calculation with modified harmonic-oscillator wave functions as given in Ref. [12] (fourth line).

\begin{tabular}{|c|c|c|c|c|c|}
\hline$m_{1} \backslash m_{2}$ & 5 & 4 & 3 & 2 & 1 \\
\hline \multirow[t]{4}{*}{5} & 0.52851 & -0.48137 & 0.37794 & -0.27795 & 0.18265 \\
\hline & 0.52857 & -0.48161 & 0.37851 & -0.27883 & 0.18355 \\
\hline & 0.53110 & -0.68046 & 0.47203 & -0.13547 & -0.07045 \\
\hline & 0.53108 & -0.61175 & 0.61258 & -0.55162 & 0.40091 \\
\hline \multirow[t]{4}{*}{4} & 0.69643 & -0.11240 & -0.11346 & 0.16657 & -0.13819 \\
\hline & 0.69619 & -0.11130 & -0.11617 & 0.17091 & -0.14264 \\
\hline & 0.45363 & -0.09097 & -0.48511 & 0.63898 & -0.31027 \\
\hline & 0.47415 & -0.11648 & -0.01808 & 0.41928 & -0.56771 \\
\hline \multirow[t]{4}{*}{3} & 0.47491 & 0.57459 & -0.30630 & 0.12814 & -0.04358 \\
\hline & 0.47557 & 0.57139 & -0.29778 & 0.11368 & -0.02814 \\
\hline & 0.41843 & 0.05608 & -0.24858 & -0.19935 & 0.62758 \\
\hline & 0.29134 & 0.25698 & -0.25966 & 0.08329 & 0.17547 \\
\hline \multirow[t]{4}{*}{2} & 0.09588 & 0.64689 & 0.47316 & -0.26552 & 0.15657 \\
\hline & 0.09470 & 0.65327 & 0.45393 & -0.22820 & 0.11179 \\
\hline & 0.34881 & 0.22208 & -0.23267 & -0.12709 & -0.00045 \\
\hline & 0.06602 & 0.38994 & -0.02755 & -0.11715 & 0.08620 \\
\hline \multirow[t]{4}{*}{1} & -0.02603 & 0.04474 & 0.69961 & 0.57982 & -0.08986 \\
\hline & -0.02477 & 0.03707 & 0.72679 & 0.51106 & 0.03469 \\
\hline & 0.28834 & 0.27451 & -0.03721 & -0.30668 & 0.09155 \\
\hline & 0.00203 & 0.13429 & 0.38065 & -0.22119 & 0.09124 \\
\hline
\end{tabular}

expression (46) as stated before. The second entry is obtained from a harmonic approximation, where the Morse wave functions are approximated by harmonic wave functions with oscillator length $a_{0}=\left[\left(j+\frac{1}{2}\right)^{1 / 2} \beta\right]^{-1}$. We see that even for the vibronic transition between the vibrational ground states in each Morse an error of the order of $10 \%$ is obtained. As soon as higher vibrational states are involved, rather important errors, as expected, occur, including a change in signs. The third entry is a calculation with modified harmonic-oscillator wave functions as given in Ref. [12]. It is seen that this calculation brings the results in the correct direction but not much. In addition it should be noted that the sum of the squares of the matrix elements in the third entry corresponding to $m_{1}=5$ are larger than 1 . This is due to the fact that the modified harmonic oscillator wave functions for each electronic state are not orthogonal.

In Table II we show a calculation in which both electronic configurations involved in the vibronic transition give rise to displaced Morse potentials with different depth and strength. In this case $j_{1}=j_{2}=5, \beta_{1}=0.90 \AA-1, R_{1}=2.67 \AA, \beta_{2}$ $=0.60 \AA^{-1}$, and $R_{2}=3.60 \AA$. The first entry here is the exact calculation. The second entry is our analytic calculation obtained by using Eq. (40). The third entry is the harmonic approximation to the wave functions. The fourth entry is the modified harmonic-oscillator approximation [12]. It can be seen that our analytic expression, Eq. (40), reproduces accurately (including signs) the exact results, with the excep- tion of the transitions involving the least bound states.

Once we have shown that the approximation given by Eq. (40) is good, we would like to apply it to a realistic system. We have chosen the stretching $\mathrm{S}-\mathrm{S}$ mode in the $\mathrm{S}_{2} \mathrm{O}$ molecule for which recently new extensive results have been reported [2]. The S-S stretching mode seems to be rather decoupled from the other modes and that allows us to consider it independently. However, we should have in mind that mixing with other modes may play a role as one goes up in excitation energy. In Fig. 1, we plot the relative dispersed fluorescence intensity versus the final state vibrational quantum number $\left(v_{1}\right)$ for three different progressions: $2_{v_{1}}^{0}(\mathrm{a})$, $2 v_{v_{1}}^{1}$ (b), and $2_{v_{1}}^{2}$ (c). ${ }^{1}$ The relative intensity is related to the

\footnotetext{
${ }^{1}$ The vibrational states in $\mathrm{S}_{2} \mathrm{O}$ are given by three labels $\left(v_{\mathrm{S}-\mathrm{O}}, v_{\mathrm{S}-\mathrm{S}}\right.$, $\left.v_{\mathrm{SSO}}\right)$ in a local basis. The label $v_{\mathrm{S}-\mathrm{O}}$ gives the number of quanta in the stretching S-O mode, the label $v_{\mathrm{S}-\mathrm{S}}$ gives the number of quanta in the stretching S-S mode, and the label $v_{\text {SSO }}$ gives the number of quanta in the bending mode. The notation for Franck-Condon transitions is $1_{v_{a}^{\prime}}^{v_{a}} 2_{v_{b}^{\prime}}^{v_{b}} 3_{v_{b}^{\prime}}^{v_{c}}$ and represents a vibronic transition between the vibrational state $\left(v_{a}, v_{b}, v_{c}\right)$ in the excited electronic configuration $\left.\widetilde{C}^{1} A^{\prime}\right)$ and the vibrational state $\left(v_{a}^{\prime}, v_{b}^{\prime}, v_{c}^{\prime}\right)$ in the fundamental electronic configuration $\left(\tilde{X}^{1} A^{\prime}\right)$. In the case in which $v_{i}=v_{i}^{\prime}=0$ the corresponding term is omitted.
} 


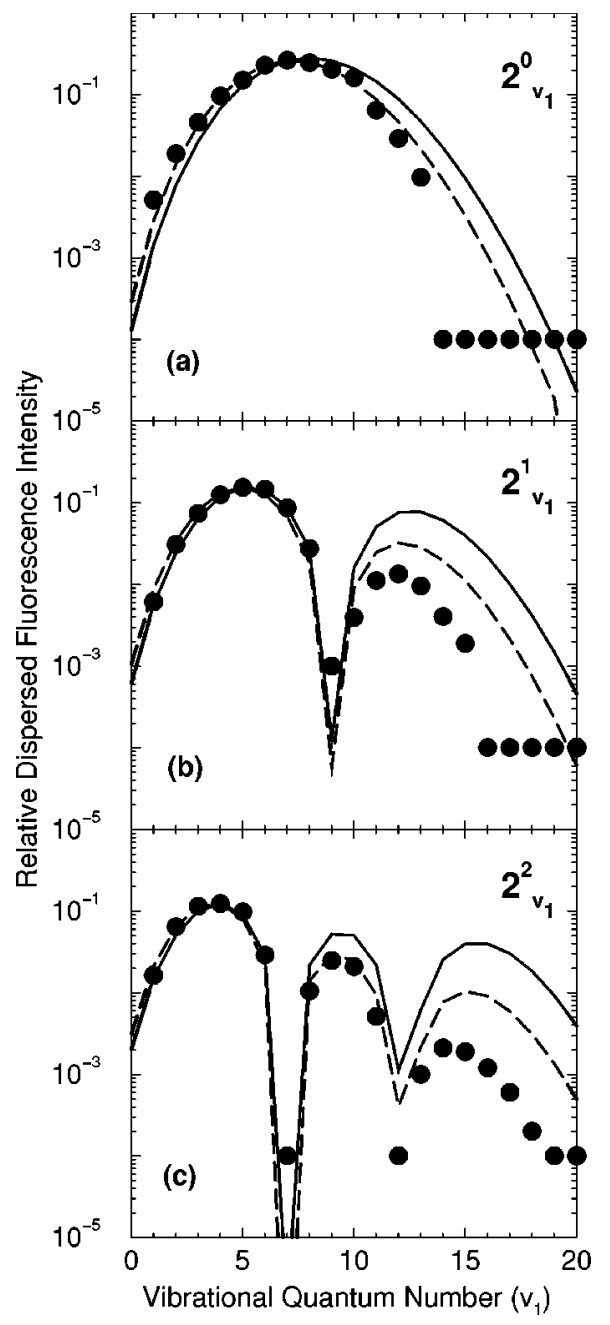

FIG. 1. Relative dispersed fluorescence intensity (dimensionless) of the stretching $\mathrm{S}-\mathrm{S}$ mode in the $\mathrm{S}_{2} \mathrm{O}$ molecule versus the final vibrational quantum number $\left(v_{1}\right)$ for three different progressions: $2_{v_{1}}^{0}(\mathrm{a}), 2_{v_{1}}^{1}$ (b), and $2_{v_{1}}^{2}$ (c). Experimental data are from Ref. [2]. Full line is the result of applying Eq. (40). Dashed line is the result of including in the overlap integral a function of $r$ (exp $[-2 \beta r]$ in this example) representing the variation of the electronic transition moment with $r$.

Franck-Condon factors $S_{\tilde{C}^{1} A^{\prime} v_{1}, \tilde{X}^{1} A^{\prime} v_{2}}$ (the square of the overlap integral) by

$$
\mathcal{I}_{\tilde{C}^{1} v_{2} \leftarrow \tilde{X}^{1} v_{1}} \propto \nu^{4} S_{\tilde{C}^{1} A^{\prime} v_{2}, \tilde{X}^{1} A^{\prime} v_{1}},
$$

where $\nu$ is the frequency of the transition and the factor $\nu^{4}$ includes the dependence $\nu^{3}$ of the spontaneous emission and an extra factor $\nu$ related to the detector response [2]. The results obtained from Eq. (40) properly normalized are plotted as solid line and the experimental data [2] as full dots. The detection limit of the experiment is $10^{-4}$. The parameters used to characterize the two Morse potentials implied in our calculation are the following: The values $j_{1}=128$ and $j_{2}=80$ are taken from Ref. [2]. The range parameters are obtained from the experimental energy of the first excited S-S stretching state in each Morse potential. In a simple approximation $\epsilon_{i}(v=1)-\epsilon_{i}(v=0)=\hbar^{2} \beta_{i}^{2}\left(2 j_{i}-1\right) / 2 \mu$. This allows us to obtain $\beta_{i}$ from the experimental energies provided the reduced mass $\mu$ is known. For the reduced mass we have accepted that, for the stretching S-S mode in the $\mathrm{S}_{2} \mathrm{O}$ molecule, the bond $\mathrm{S}-\mathrm{O}$ is rather stiff and the reduced mass can be evaluated as $\mu=M_{\mathrm{S}} M_{\mathrm{SO}} /\left(M_{\mathrm{S}}+M_{\mathrm{SO}}\right)$. With this approximation the values $\beta_{1}=1.744 \AA^{-1}$ and $\beta_{2}=1.718 \AA^{-1}$ are obtained. From the experimental data the equilibrium S-S separation in the electronic ground state is known to be $R_{1}$ $=1.8845 \AA$ and for the equilibrium separation in the electronic excited state it is $R_{2}=R_{1}+\Delta R$, with $\Delta R=0.26 \AA$ [17] obtained from an analysis of the rotational structure of the $\mathrm{S}_{2} \mathrm{O}$ molecule. With this input we have calculated the fluorescence intensity, which is presented in Fig. 1. It can be seen that the main structure of the relative intensity in all cases is reproduced correctly, although it is overestimated for large final vibrational quantum numbers $\left(v_{1}\right)$. One reason for this disagreement is the fact that we are treating the stretching S-S mode as isolated. This should be a good approximation for small values of $v_{1}$ but mixing with the other modes in the molecule is expected to be more important as $v_{1}$ increases. Another reason could be the calculation of the Franck-Condon factor by using the usual approximation of replacing the electronic transition moment, which should be a function of the internuclear separation $r$, by a constant. This may not be a good approximation when considering vibrational states with large $v_{1}$. In that case the electronic transition moment written as an appropriate function of $r$ should be included in the overlap integral, such as presented in Eq. (32). We have performed a calculation of this type just to show the effect of such a term. The electronic transition moment has been taken as proportional to $\exp [-2 \beta r]$. The result of this calculation is shown in Fig. 1 with a dashed line. It is seen that a term of this kind moves all the results in the correct direction, although, of course, it is not able to correct all discrepancies at large $v_{1}$ since mixing should also be important.

\section{SUMMARY AND CONCLUSIONS}

In this paper we present an approximate analytic expression to evaluate Franck-Condon overlap integrals for anharmonic wave functions. This expression is based on the Gaussian method of integration by quadratures and on the recently introduced configuration localized Morse states. The expression is shown to be exact for Morse wave functions with the same range parameter $\beta$ and different values of the equilibrium position $\left(R_{1}\right.$ and $\left.R_{2}\right)$ and depths $\left(j_{1}\right.$ and $\left.j_{2}\right)$ and it is a very good approximation for general Morse wave functions. In particular it has been applied to the S-S stretching mode in the $\mathrm{S}_{2} \mathrm{O}$ molecule leading to a correct description of the experimentally observed fluorescence intensities. In addition, it has been shown that the removal of the Condon approximation so as to include explicitly the dependence of the electronic transition dipole moment on the internuclear distance, which is straightforward in our treatment, improves the description of the experimental data.

We consider that the approximation presented here constitutes a useful starting point in order to obtain analytical approximations for Franck-Condon factors in polyatomic molecules. In that case it can be used in connection with the algebraic models recently developed in terms of the su(2) algebra, which take into account the interactions between different local modes of the molecules, and provide finally 
the wave functions as combinations of products of algebraic local wave functions. These algebraic local wave functions can be written in a coordinate representation as Morse wave functions, or possibly Pöschl-Teller wave functions for the bending modes. The formalism presented here can be used then to calculate the overlap integrals. In relation with this last point, it is worth mentioning that the approximations presented here can be extended to other anharmonic potentials of interest in molecular physics, such as, for example, the Pöschl-Teller potential, which has been suggested to be more appropriate for representing bending modes in polyatomic molecules.

\section{ACKNOWLEDGMENTS}

This work was supported in part by the Spanish DGICYT under Project No. PB95-0533. We acknowledge useful dis- cussions with F. Pérez-Bernal, A. Frank, and R. Lemus.

\section{APPENDIX}

In this appendix we are showing that Eq. (46) is equivalent to the form recently reported in Ref. [12] for Morse wave functions of potentials with the same range $\beta$. We start from Eqs. (46). We will use Eqs. (42)-(44) and the explicit expression of the generalized Laguerre polynomials,

$$
L_{n}^{(\alpha)}(y)=\sum_{\ell=0}^{n} \frac{(-1)^{\ell}}{\ell !}\left(\begin{array}{c}
n+\alpha \\
n-\ell
\end{array}\right) y^{\ell}
$$

Taking into account the relation between our notation and the one used in Ref. [12], $N=2 j, n=j-m, \zeta=c_{1} / c_{2}$, the previous equation, and Eq. (7), we can write Eq. (46) as

$$
\begin{aligned}
I= & \mathcal{N}_{N_{1}, n_{1}}^{-1 / 2} \mathcal{N}_{N_{2}, n_{2}}^{-1 / 2} \frac{c_{1} c_{2}}{c^{2}} \sum_{k=1}^{j} N_{k}^{-1}\left[\frac{c_{1}}{c} y_{k}\right]^{\left(N_{1} / 2\right)-n_{1}-1}\left[\frac{c_{2}}{c} y_{k}\right]^{\left(N_{2} / 2\right)-n_{2}-1} \sum_{1=0}^{n_{1}} \frac{(-1)^{\ell} 1}{\ell_{1} !}\left(\begin{array}{c}
N_{1}-n_{1} \\
n_{1}-\ell_{1}
\end{array}\right) \\
& \times\left[\frac{c_{2}}{c} y_{k}\right]^{\ell} \sum_{\ell_{2}=0}^{n_{2}} \frac{(-1)^{\ell} 2}{\ell_{2} !}\left(\begin{array}{c}
N_{2}-n_{2} \\
n_{2}-\ell_{2}
\end{array}\right)\left[\frac{c_{2}}{c} y_{k}\right]^{\ell} \cdot
\end{aligned}
$$

Since $c_{1} / c=[2 /(1+\zeta)] \zeta$ and $c_{2} / c=2 /(1+\zeta)$, we can rewrite the preceding equation as

$$
\begin{aligned}
I= & \mathcal{N}_{N_{1}, n_{1}}^{-1 / 2} \mathcal{N}_{N_{2}, n_{2}}^{-1 / 2} \zeta \frac{N_{1}}{2}-n_{1}\left(\frac{2}{1+\zeta}\right)^{\left(N_{1}+N_{2} / 2\right)-n_{1}-n_{2}} \sum_{\ell_{1}=0}^{n_{1}} \sum_{\ell_{2}=0}^{n_{2}} \frac{(-1)^{\ell}+\ell_{2}}{\ell_{1} ! \ell_{2} !}\left(\begin{array}{l}
N_{1}-n_{1} \\
n_{1}-\ell_{1}
\end{array}\right)\left(\begin{array}{l}
N_{2}-n_{2} \\
n_{2}-\ell_{2}
\end{array}\right) \zeta \ell_{1}\left(\frac{2}{1+\zeta}\right)^{\ell}+\ell_{2} \\
& \times \sum_{k=1}^{j} N_{k}^{-1} y_{k}^{\ell} \ell^{+} \ell_{2}+\left(N_{1}+N_{2} / 2\right)-n_{1}-n_{2}-2
\end{aligned}
$$

Remembering that the index $k$ labels the zeros of the generalized Laguerre polynomial $L_{j}^{(1)}(y)$, the expression of the Gaussian integration by quadratures, Eqs. (21), (22), and the definition of the $\Gamma$ function, the sum on $k$ can be written as

$$
\sum_{k=1}^{j} N_{k}^{-1} y_{k}^{\ell}+\ell_{2}+\left(N_{1}+N_{2} / 2\right)-n_{1}-n_{2}-2=\int_{0}^{\infty} d y y \exp [-y] \ell_{1}+\ell_{2}+\left(N_{1}+N_{2} / 2\right)-n_{1}-n_{2}-2=\Gamma\left(\frac{N_{1}+N_{2}}{2}-n_{1}-n_{2}+\ell_{1}+\ell_{2}\right) .
$$

Substituting this last result in the expression of $I$, the final result is obtained,

$$
\begin{aligned}
I= & \mathcal{N}_{N_{1}, n_{1}}^{-1 / 2} \mathcal{N}_{N_{2}, n_{2}}^{-1 / 2} \zeta^{N_{1}}-n_{1}\left(\frac{2}{1+\zeta}\right)^{\left(N_{1}+N_{2} / 2\right)-n_{1}-n_{2}} \sum_{\ell_{1}=0}^{n_{1}} \sum_{\ell_{2}=0}^{n_{2}} \frac{(-1)^{\ell}+\ell_{2}}{\ell_{1} ! \ell_{2} !}\left(\begin{array}{c}
N_{1}-n_{1} \\
n_{1}-\ell_{1}
\end{array}\right)\left(\begin{array}{c}
N_{2}-n_{2} \\
n_{2}-\ell_{2}
\end{array}\right) \zeta^{\ell}\left(\frac{2}{1+\zeta}\right)^{\ell_{1}+\ell_{2}} \\
& \times \Gamma\left(\frac{N_{1}+N_{2}}{2}-n_{1}-n_{2}+\ell_{1}+\ell_{2}\right)
\end{aligned}
$$

which is precisely Eq. (4.5) in Ref. [12].

[1] G. Herzberg, Molecular Spectra and Molecular Structure: I. Spectra of Diatomic Molecules (Van Nostrand Reinhold, New York, 1950).

[2] T. Müller, P. Duprè, P. H. Vaccaro, F. Pérez-Bernal, M. Ibra- him, and F. Iachello, Chem. Phys. Lett. 292, 243 (1998).

[3] T. E. Sharp and H. M. Rosenstock, J. Chem. Phys. 41, 3453 (1964).

[4] A. Warshel and M. Karplus, Chem. Phys. Lett. 17, 7 (1972); 
A. Warshel, J. Chem. Phys. 62, 214 (1975).

[5] E. V. Doktorov, I. A. Malkin, and V. I. Man'ko, Chem. Phys. Lett. 46, 183 (1977); J. Mol. Spectrosc. 64, 302 (1977); 77, 178 (1979).

[6] W. Domcke, L. S. Cederbaum, H. Köppel, and W. von Niessen, Mol. Phys. 34, 1759 (1977); H. Kupka and P. H. Cribb, J. Chem. Phys. 85, 1303 (1986).

[7] J. F. Rivas-Silva, G. Campoy, and A. Palma, Int. J. Quantum Chem. 43, 747 (1992).

[8] R. W. Nicholls, J. Chem. Phys. 74, 6980 (1981).

[9] P. J. Drallos and J. M. Wadehra, J. Chem. Phys. 85, 6524 (1986)

[10] P. M. Morse, Phys. Rev. 34, 57 (1929).
[11] G. Pöschl and E. Teller, Z. Phys. 83, 143 (1933).

[12] F. Iachello and M. Ibrahim, J. Chem. Phys. A102, 9427 (1998).

[13] E. Ley-Koo, S. Mateos-Cortés, and G. Villa-Torres, Int. J. Quantum Chem. 56, 175 (1995), and references therein.

[14] M. Carvajal, J. M. Arias, and J. Gómez-Camacho, Phys. Rev. A 59, 1852 (1999).

[15] M. Berrondo and A. Palma, J. Phys. A 13, 773 (1980).

[16] Handbook of Mathematical Functions, edited by M. Abramowitz and I. Stegun (Dover, New York, 1972).

[17] K. E. Hallin, A. J. Merer, and D. J. Milton, Can. J. Phys. 55, 1858 (1977). 